\title{
Still Striving After All These Years: Between Normality of Conduct and Normativity of Evaluation in Casual Relationships among College Students
}

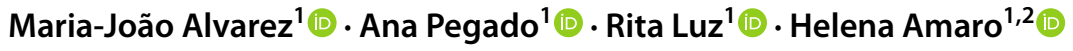 \\ Accepted: 26 September 2021 \\ (c) The Author(s), under exclusive licence to Springer Science+Business Media, LLC, part of Springer Nature 2021
}

\begin{abstract}
Gender similarities have been detected in various sexual behaviors and attitudes in young adults, but persistent differences remain regarding casual sexual relationships (CSRs), with women feeling different external pressures than men. We set out to study the spontaneous references made to gender similarities and differences towards CSRs in eight focus group interviews ( $N=35$ college-students, aged 18-28) about the characteristics of CSRs within a social-role framework. The thematic analysis led us to the interpretation that there is an ongoing change in the acceptability of these relationships, leading to the emergence of a single sexual standard - mostly liberal, but at times also conservative. However, different expectations remain, derived from a deep-rooted traditional sexual double standard still perceived in society. Challenges to the unequal standards and social constraints in CSRs is desired by many women, defended by men, and admired in those women whose striving contributes to the normalization of this conduct and of how it is evaluated.
\end{abstract}

Keywords Young adult $\cdot$ Focus group $\cdot$ Sexual behavior $\cdot$ Casual sex $\cdot$ Gender equity $\cdot$ Gender differences

Meta-analytic reviews on gender differences in sexuality have shown within-gender variation in sexual behaviors and attitudes to be larger than between-gender variation; while men reported more sexual behaviors and more permissive sexual attitudes, the differences were small (Petersen \& Hyde, 2010, 2011). A recent meta-analysis assessing differences in people's expectations and judgments regarding sexual behavior concludes that there is evidence for a traditional Sexual Double Standard (SDS) (a dual set of sexual standards that is stricter for women than for men) - though the effect is small, and this evidence depends on the methodology used for assessing the SDS (Endendijk et al., 2020). This meta-analysis, like previous reviews of the literature (Bordini \& Sperb, 2013; Petersen \& Hyde, 2010, 2011), showed that the traditional SDS is behavior-specific, indicating that certain behaviors no longer elicit the SDS, while

Maria-João Alvarez

mjalvarez@psicologia.ulisboa.pt

1 Faculdade de Psicologia, Universidade de Lisboa, CICPSI, Alameda da universidade, 1649-013 Lisboa, Portugal

2 Faculdade de Psicologia E de Ciências da Educação, Universidade de Coimbra, Lisboa, Portugal others, such as casual sex, continue to be differently accepted when performed by a man or a woman. Casual sex may be defined as involving various forms of sexual behavior, without there being mutual expectations of romantic involvement or that the relationship will be ongoing, with the duration and degree of knowledge between individuals being highly variable (Alvarez et al., 2019). Other moderators for SDS include the cultural background, and the bidimensional nature of the concept, with the social representation of the norm shown to be more traditional than personal attitudes are. It can therefore be said that evidence reveals a convergence on sexual attitudes and behaviors denoting gender equality, but certain results remain entrenched, namely, the recognition and, in some cases, the adoption of differential gendered norms toward casual sex, in various ways relative to cultural differences.

In this article we argue that emphasis on gender differences instead of gender similarities tends to perpetuate double standards. While many sexual behaviors no longer elicit the SDS, others that continue to be the target of gendered judgments, such as casual sex, are culturally dynamic sexual realities that also undergo changes that are important to monitor and understand. We begin by presenting reasons for the perpetuation of the SDS in casual sex and the social 
game in which young women must navigate between resistance and accommodation. We show the social relevance and normative role of CSRs and provide a brief cultural account of the behaviors that characterize the Portuguese sexual context. We then discuss empirical research showing sexual agency in women in casual sex. Finally, we report our study based on the spontaneous allusions made to gender similarities and differences in CSRs in focus group interviews with female and male college students in this less studied culture.

\section{The Sexual Double Standard in Casual Sex}

Differential evaluation of casual sex demonstrates the perpetuation of the SDS; this may be attributed to genderrelated differences ingrained in the social structure, specifically the concentration of men and women in different social roles, as argued by the social role theory (Eagly \& Wood, 1999). While male-dominated roles tend to be characterized by greater power and higher status, female-dominated roles are associated with subordinate behaviors, accounting for a gender hierarchy that also reflects on sexual behavior, attributing more sexual control to men and less sexual autonomy to women (Eagly \& Wood, 1999). From this, there result traits that characterize men (agency) and women (communion) - gender role beliefs and stereotypes - that tend to be considered desirable and appropriate for each sex. Conformity to gender role expectations is rewarded, and deviance is negatively evaluated or punished (e.g., Wood \& Eagly, 2012).

Despite gender roles having become less strict in most Western societies (Eagly \& Wood, 1999), which points toward personal attitudes and societal expectations being marked by greater gender equality in the acceptance/judgment of sexual conduct, women continue to feel different external pressures regarding sex than men do, as if sexual double standards have been shifted away from pre-marital sex, sexual practices, and infidelity, toward casual sexual relationships (CSRs). The existence of these areas of exception can be understood as a way to maintain the status quo of women's subordination to certain gender role beliefs and stereotypes-materialized in the idea that an active, detached sexuality is acceptable or even expected for men, but not for women-and the meaning attributed to such areas of exception has not been sufficiently explored in different cultural contexts.

In fact, studies continue to find that sex in hookups is more controversial for women than for men and more status-producing for men than for women (England \& Bereak, 2014), with more permissiveness of casual sex granted to men; this attitude is held more strongly among men than among women (Sprecher et al., 2013), although gender was not found as a moderator in the evaluation or expectations of men's and women's sexual behavior more recently (Endendijk et al., 2020). Rather than endorsing the SDS, women appear to recognize it as a social norm and feel they must conform with it - or make others believe they do - in order to protect their sexual reputations (Fjaer et al., 2015). Women's discourses alternate between resistance - viewing the SDS as sexist injustice - and accommodation - concerns about one's reputation and silence about casual encounters (Farvid et al., 2017), indicating the simultaneous adoption of an egalitarian standard as well as enactment of the SDS. The strength of the SDS therefore lies in the fact that it is a socially recognized standard (social SDS) that has been maintained over the years, in spite of its weakening as a personally internalized standard (personal SDS) (Endendijk et al., 2020). Alternative standards have been adopted (personally) - an egalitarian single sexual standard (SSS), with the same level of permissiveness or restrictiveness applied to sexual behavior of both men and women - and there is also residual evidence of a reversed sexual double standard, with more restrictiveness applied to male than female sexual behavior (Bordini \& Sperb, 2013; Endendijk et al., 2020). This seems to be the case in Portugal, where research, although limited, has shown that both genders tend to behave similarly; concerning the judging of others, young women adopt egalitarian and sometimes reversed standards (Ramos et al., 2005), and young men show an attenuated SDS (Marques et al., 2013), but both recognize the social SDS or assume that pressure exists for men and women to conform with the prescriptions of the traditional standards (Amaro et al., 2021; Ramos et al., 2005). The effects of this inconsistency around behaviors that are the new targets of the SDS, such as casual sexual relationships, have not been explored in Portuguese studies.

Even though women see themselves as more agential in actual sexual encounters than how men tend to view them, this being attributed to women's self-concepts having become increasingly agential over time (Eaton et al., 2016), gender roles persist in casual sexual scripts showing gender expectations and stereotypes enacted within these relationships (Eaton et al., 2016; Olmstead et al., 2019). Reid et al. describe the uncertain game navigated by young women: "While hookups can offer women sexual pleasure without the demands of relationships, they occur within the gendered context of an ongoing sexual double standard" (2011, p. 548).

\section{The Social Relevance of CSRs}

The possibility of CSRs being an area where the struggle for equality takes place - insofar as they are at the service of the maintenance of the SDS and the resultant inequalities - is particularly important as the number of people involved 
in CSRs has grown in the last two decades (Alvarez et al., 2019; Garcia et al., 2012; Wentland \& Reissing, 2011). Such an increase may be attributed both to individuals prioritizing specific life tasks - finishing university, being financially independent, starting and investing in the development of a (professional) career - and to deferring family formation, which Hamilton and Armstrong (2009) have termed the self-development imperative. Gender equity - the increase of women's participation in higher education and in the labor force - is among the main reasons why the number of single individuals has increased (e.g., Himawan et al., 2018). The prioritization of these tasks therefore crosses diverse cultures (e.g., Arnett, 2015; Himawan, 2020) and is not limited to men, having changed most of all in women; it allows emerging adults to allocate resources to academic and professional advancement, while living their sexuality without compromising their independence. Freedom of action and lifestyles is one of the merits of single life most frequently cited by both men and women in many cultures (e.g., Lyons et al., 2014; National Institute of Population \& Social Security Research, 2017).

Portuguese society in the 1960s was configured around a strict conservative structure in matters of sexual morality, following the orientation of the Catholic Church. As has happened in many Western societies, major changes have since taken place in social structure and sexual behavior. Portugal today has more women finishing higher education and with doctorates than men (PORDATA, 2021). Whereas in 1990, the average age at marriage was 26.2 years for men and 24.2 years for women, these figures were 33.9 and 32.4 years in 2019, respectively, and the average age at first childbirth rose from 24.7 years for women in 1990 to 30.5 years in 2019 . Getting married and/or starting a family continue to be projects, but are envisioned in the distant (Machado et al., 2014). Despite $81 \%$ of the population being Catholic, suggesting a religious endorsement to marry, there were more people married in civil ceremonies $(68.7 \%)$ than married by the church (30.7\%) in 2019 (PORDATA, 2021). This more secular and less-valued view of the sacramental and institutional aspect of marriage does not reflect a rejection of it, but is the result of the experience of various relational alternatives before marriage, since most of the experiences of informal conjugality prove to be transitory (Aboim, 2005). From the sexual point of view, there was a convergence of male and female sexual behavior, especially among the young, with major changes among women, especially those with higher education (Ferreira \& Villaverde, 2010). However, research focused on understanding the gendered diversity of responses to dominant (hetero)sexual scripts has showed the coexistence of still-conservative discourses alongside others that are more progressive. The progressive ones strive to detach from the traditional frames and gender rules for relationships and sex, via increasing sexual agency, and in some cases stepping outside of traditional gender roles by adopting new forms of intimate relationship or by opting for (Alarcão et al., 2015).

It can therefore be noted that important contributions to the increase of sexual relationships during emerging adulthood in Portugal include: academic and professional investment and the subsequent postponement of marriage and having children, the acceptance of female sexuality outside marriage and the dissolution of sexual prohibitions, the lesser impact of religion on behavior, and the expectation of different ways of experiencing intimacy before marriage.

\section{Sexual Agency in Women in Casual Sex}

Gender differences in sexuality have proven to be smaller in societies with greater gender equality (Endendijk et al., 2020; Petersen \& Hyde, 2010) and researchers argue that focus should be placed on gender similarities rather than differences, which tend to perpetuate double standards when emphasized (Hyde, 2005). One such similarity concerns positive attitudes towards postponing marriage or remaining single, which is found across many cultures (Himawan et al., 2018) and which increases the likelihood and the relevance of non-committed sexual relationships.

In fact, acceptability for women's sexual agency in hookups has also been found, with women seen as sexual beings with physical desires at the basis of their hookup behavior and with college students of both genders recognizing the normalcy of women's sexual desire and the right to pursue sex in a hookup without the expectation of a relationship (Reid et al., 2011). In addition, both genders were perceived as having convergent objectives of rapport-building when refraining from sex on a post-hookup date, far from the approach/avoidance dichotomy usually assigned to the genders (Reid et al., 2011). This acceptability is not devoid of some caveats, especially in a post-hookup date; a woman is more perceived as needing to improve her self-image and to show herself as a potential dating partner (Reid et al., 2011). Rudman et al. (2017) showed that when the costs of involvement in casual sex were mild, the difference by which men received greater encouragement was eliminated, and when the costs were higher for men, women were urged by their fellow participants to have casual sex more than men were. This suggests that women may receive equal encouragement to get involved in casual sex, depending on the risk involved rather than on their gender. Besides this liberal egalitarian sexual standard, other recent studies found some evidence of a single conservative sexual standard for casual sex behavior among men and women, with mainly conservative gender egalitarianism as the norm (Allison \& Risman, 2013). These changes sometimes developed toward a reverse sexual double standard, with men being judged more harshly 
and seen as less appealing for being 'sluts' than the female 'sluts' (Papp et al., 2015). Finally, it should be noted that predictors of gender equality in sexual regret from casual sex include factors that are mainly situational, such as being the one who takes the initiative, finding the partner sexually competent, and sexual gratification (Kennair et al., 2018); this shows that the differences may be diluted, given the possibility of higher agency and some luck when choosing the partner. In sum, several gender similarities have been found regarding the experience of CSRs, in terms of their acceptability, the need for rapport-building in some of these encounters, and social encouragement in situations where risks are controlled; even regret from casual sex is shown to be more dependent on situational aspects than on gender.

In the field of sexuality there are significant indications that gender similarities are greater than usually emphasized in the studies and that, even in domains where differences persist, such as in CSRs, sexuality is dynamic and is constantly being renegotiated. It is also the case with CSRs that more similarities are found than are usually emphasized, and this relational domain should therefore be continuously monitored so that we remain aware of the ongoing changes and their implications for gender equality. The purpose of the present study, which is part of a larger study on the characteristics of CSRs, was to analyze spontaneous allusions made to gender similarities and differences in CSRs, without being prompted by emphasis on the differences, in focus group interviews with college students in a less-studied cultural context.

\section{Materials and Methods}

\section{Participants}

Participants comprised 35 college students $(M=20.89$; $S D=2.17), 19$ women, mostly heterosexual (cf. Table 1).

Participants were recruited through a convenience sampling method from five Portuguese universities from different regions of the country; the study was presented to students during classes and those interested were pre-enrolled to participate; inclusion criteria included to be between 18 and 29 years of age, speaking European Portuguese as their native language, and having had at least one sexual experience. Living in the country for less than a year was an exclusion criterion as it was considered to imply an insufficient command of the language.

\section{Procedure}

We presented the study as seeking to know and deepen our knowledge about the CSRs that Portuguese emerging adults engage in today. Seventy-two students pre-enrolled,
Table 1 Sociodemographic Characteristics of Participants $(\mathrm{N}=35)$

\begin{tabular}{|c|c|}
\hline \multicolumn{2}{|l|}{ Participants' characteristics } \\
\hline Age range & $18-28$ \\
\hline Gender & $54 \%$ Women, $46 \%$ Men \\
\hline Ethnicity & $\begin{array}{l}\text { 94\% White } \\
3 \% \text { African-European } \\
\text { 3\% Latino }\end{array}$ \\
\hline Religious affiliation & $54 \%$ \\
\hline \multicolumn{2}{|l|}{ Sexual experiences } \\
\hline $\begin{array}{l}\text { Sexual interactions only with the other } \\
\text { sex }\end{array}$ & $80 \%$ \\
\hline $\begin{array}{l}\text { Sexual interactions mainly with the other } \\
\text { sex }\end{array}$ & $8.6 \%$ \\
\hline $\begin{array}{l}\text { Sexual interactions only with the same } \\
\text { sex }\end{array}$ & $5.7 \%$ \\
\hline Sexual interactions with both sexes & $5.7 \%$ \\
\hline \multicolumn{2}{|l|}{ Relationship status } \\
\hline Single & $30.3 \%$ \\
\hline In a committed relationship & $66.7 \%$ \\
\hline Non-marital partnership & $3 \%$ \\
\hline \multicolumn{2}{|l|}{ Previous CSR experience } \\
\hline No CSR experience & $25.7 \%$ \\
\hline $1-3$ CSRs & $28.5 \%$ \\
\hline 4-6 CSRs & $22.8 \%$ \\
\hline 7-9 CSRs & $8.5 \%$ \\
\hline$>10 \mathrm{CSRs}$ & $14.5 \%$ \\
\hline \multicolumn{2}{|l|}{ Number of partners } \\
\hline 1-3 partners & $42.8 \%$ \\
\hline 4-6 partners & $28.7 \%$ \\
\hline 7-9 partners & $11.5 \%$ \\
\hline$>10$ partners & $17.4 \%$ \\
\hline
\end{tabular}

and thirty-six were scheduled to focus groups according to their best convenience. Concerning those who did not participate, the main reasons were being unavailable on the proposed schedules, not responding to the initial e-mails, and not completing the scheduling procedure; one student could not participate because she was living in the country for less than a year, and another stated no longer being interested in participating. In the case of the online focus group, two participants were unable to guarantee privacy, and one scheduled participant arrived late and could no longer join the Zoom meeting to participate in the focus group interview.

Ethical approval was granted by the authors' faculty review board (n.1_2017_18), and all participants signed an informed consent statement. All students received a gift check $(10 €)$ in return for their participation.

Four same-gender (two female and two male) and four mixed-gender focus groups were performed, with 3-6 participants per group. Interviews took from 60 to $110 \mathrm{~min}$, and were all conducted by the third author, already experienced in conducting group interviews. All focus groups took 
place between November and December 2019 in the higher education institutions where the participants were recruited, except for one which took place through the Zoom platform in April 2020, due to restrictions imposed during the Covid19 pandemic.

This study was part of a wider research project aiming to explore the variation and characteristics of casual sexual relationships among Portuguese young adults. The decision to conduct focus-group interviews was taken in order to reach in-depth information about the socially-shared perceptions and opinions about CSRs rather than personal and individual experiences, as well as to use the discussion to find the most typical language, terms, and expressions used by Portuguese emerging adults when talking about CSRs (Frith, 2000).

We created a semi-structured interview guide aimed at deepening the characterization of the different types of CSR. The questions were based on previous research finding a variety of CSRs (Alvarez et al., 2021) and were informed by the literature on CSRs (e.g., Wentland \& Reissing, 2011). In order to initiate and facilitate communication between participants and moderator, a neutral question was first posed to all participants(e.g., about each participant's area of study), followed by a general question about what CSRs are and their main characteristics, then key-questions about specific features of CSRs (motivations, beginning and ending of an encounter, communication, scripts, conflict management, thoughts and feelings, sexual experience, benefits and risks, transitions, safe sex issues, the role of new technologies, and social acceptance). For each question participants were asked to share their views of socially-held opinions and not their personal experiences, although they could do so if they wished. Probes, follow-ups, and unscripted questions were presented by the moderator in order to obtain further details (Krueger, 1998). Although some questions were meant to explore the social acceptance of casual sex, none referred to gender differences nor directly examined the existence of SDS. To conclude the interview, the moderator presented a brief summary of the main contributions and invited participants to comment.

After being interviewed, participants completed a sociodemographic questionnaire (see Table 1). Participants in the online focus group completed the questionnaire using an online platform (Qualtrics).

\section{Analysis Procedures}

All interviews were conducted in Portuguese, audio-recorded and transcribed verbatim. In order to identify and interpret the socially-produced patterns of information across data, we performed a thematic analysis of data, inspired by the guidelines of Braun and Clarke (2006). In a first step, using NVivo 12 software, one researcher familiarized with data and identified, selected and compiled all excerpts containing spontaneous allusions to gender similarities and differences in CSRs, as well as discourses around the words 'man', 'woman', 'men', 'women', 'boy(s)', 'girl(s)', 'gender', 'equality', and 'inequality'. In a second step, a different researcher reread all the excerpts and attributed initial codes to individual quotes that captured participants' reflections about gender. The codes were descriptors of the content of each quote about gender-related issues in CSRs. A third researcher reread the quotes to validate the attributed codes or propose alternatives. These researchers jointly resolved what divergences there were and, in a third step, analyzed and combined the initial codes to form broader themes. Subsequently, all authors reviewed the initial thematic map in a collaborative and recursive process, and delineated the final set of themes for gender similarities and differences. This analytical process resulted in two main themes and five sub-themes. We followed the COREQ checklist (Tong et al., 2007) to report the main characteristics of this qualitative study (Supplementary file), explicitly and comprehensively.

\section{Results}

All participants had already had intercourse; $74 \%$ reported at least one casual sex relation at some time in life, with $14.3 \%$ being currently involved in one; the mean number of their casual sexual partners so far was 7.6 (Table 1).

Two themes were generated from the analysis of the interviews: The Single Sexual Standard and the Sexual Double Standard. Excerpts identify gender (W for woman, $M$ for man), participant (through a number after gender identification), and the type (sg for single-gender or $\mathrm{mg}$ for mixedgender) and number of the focus group.

\section{The Single Sexual Standard}

\section{Social Acceptance}

Most of the participants considered that CSRs are accepted within their generation since '(...) society has an increasingly open-minded mentality' (M4_sg2). In this sense, young men considered that 'It is important for anyone to pursue the (sexual) practices they want without being judged for it' (M2_sg8), with no gender-related exception because '(...) girls have exactly the same right that we do (...) (M3_sg2). It's not even the right, it's the same desire, we're humans' (M2_sg2). Young women also claimed the same right to have casual sexual partners and to brag about it, 'Go girl! Do what you gotta do! (...) If that's what you want, it's your body...' (W2_sg6). These statements point towards advances in the depenalization of CSRs, leading to increasing gender equality. 
Participants recognized that women's sexual agency and orientation towards their own satisfaction have become more prominent, as nowadays women feel that they '(...) are taking advantage of this boom of information and openness' (W1_mg3) and so 'There are more women that search only for casual and physical [pleasure]' (W1_sg6). A number of young women stated that they feel comfortable in initiating and leading a CSR, whereas others mentioned that women are increasingly taking preemptive action regarding sexual protection, 'I see more girls carrying condoms than boys' (W1_sg5). One female participant recounted one of her personal experiences where sex was the most important part in a relationship, '(...) I let myself be carried away and... in [each of] the three times we began the encounter with sex' (W1_mg4).

\section{Favorable Contexts}

Participants also expressed that some specific circumstances could help minimize the impact of social judgment about involvement in CSRs. The fact that some young adults are studying far away from home creates a safer space to explore sexuality because 'It helps, the fact that since no one knows you, you are more at ease to do as you please' (W2_sg5). Along with a new space of freedom, there also appears to be a "defined" time to experience CSRs, reflecting similar gender perspectives. Both men and women explained that they participate in casual relationships because they want to have enjoyable uncommitted sexual relationships, and they also stated a common long-term goal of having '(...) a [serious] relationship, not only casual things (...)' (M2_sg2). Young women also endorsed this aim, stating that 'In my opinion, we'll get to a point where we'll be tired of... (W2_sg5) ... always being in casual relationships' (W3_sg5). 'We'll get to a point where we'll want something more, we'll want someone with whom to... do other stuff than going to parties or...(W2_sg5) [Someone with whom to] share other experiences!' (W4_sg5).

\section{Limits, Difficulties, and Challenges}

Participants also commented on some of the limits and difficulties that both women and men face when dealing with CSRs. Their discourses stressed that there has to be a limit of partners at some point, determined either by a number or by the arrival of a certain moment in life. As one male participant said, '(...) aren't you disrespecting yourself, whether you are a man or a woman (...) being with 10 people in one night, kissing 10 mouths in the same night, don't you feel a little disrespected?' (M3_mg1). Having too many partners seemed to call into question not only women's but also men's reputations, in the sense that ' $(. .$.$) we [men] are differently$ criticized, if a man has already been with, I don't know, 15 girls, 20 girls, [they say] that this one is only good for that, that he will never have a serious relationship. (...) And that pushes girls away' (M4_mg3). This egalitarian conservative judgment is closer to their parents' values, as stated by two male participants who stressed that their mothers reproached them for having too many female sexual partners and not settling down with only one person.

When it comes to initiating and leading a CSR, participants accorded more importance to psychological variables than to gender: '(...) Nowadays it's the person that has more self-esteem or has a bigger ego - whoever feels more confident in that specific situation is the one who's going to lead [the encounter], whether it is the man or the woman...' (M3_mg1), pointing to some equality in this aspect. However, some gender-specific difficulties were mentioned concerning engaging in casual sex. For example, a number of male participants affirmed that men feel that sexual encounters are difficult to initiate, which is why '(...) drinking is very important (...) knowing that I'm going to be with a girl, or that I'm going to have sex with a girl, if I'm not a bit drunk, I don't have the guts to start a conversation' (M3_sg2). They also mentioned that, from their perspective, (casual) sex may be more uncomfortable for women than for men because of ' $(. .$.$) the shame of showing their own$ bodies or even looking at [men's] bodies and not wanting to show their own' (M3_sg2). For female participants, while recognizing that women's sexual behaviors are progressively guided by their willingness to experience pleasure, the main difficulty seemed to be the still-uncertain social acceptance of women's involvement in CSRs, because although 'Women also think a lot about pleasure' (W3_sg5), they also must ' (...) have the capacity not to care about what is said' (W2_sg6).

\section{The Sexual Double Standard}

\section{Social Constraints}

The observation that ' $(.$. ) in our generation (...) a boy that goes out with lots of girls (...) is a champion, and a girl that goes out with lots of boys (...) is a slut (...)' (M4_sg2), showed the perception that society continues to hold a biased perspective on the acceptability of CSRs. Our participants, both women and men, felt the need to stress that there are different expectations towards each gender, through distinct gender roles that include sexual behaviors. These young adults mentioned that there is no definite rule regarding the number of sexual partners for men, while for women it is something like 'One every six months, and...! More than that, and they're considered sluts!' (W2_mg4). 
Social conventions still make the rules, as some women admitted that most of the times when they refrain from taking the initiative for CSRs it is because it may destroy a future committed relationship as 'For casual, [men] like easy [women], but for a committed relationship...' (W1_ sg6). On the other hand, social pressures also call for a sense of obligation to have a busy sex life, especially (but not exclusively) for men, 'Yes, there is a pressure from people around us that if we are not involved sexually with someone we are perceived as a failure...' (W3_mg3).

\section{Some Women Judge, other Women Flourish}

Contributing to this SDS may be not only the traditional beliefs about gender, but also the fact that ' (...) women (...) are the first to judge other women for something that, probably, we also do' (W2_sg5). Most female participants were well aware of this criticism and affirmed that they use it at times when they want to hurt someone, 'I think that we don't have many problems with that, but when we want, we use it against the person' (W1_mg7). There was also an opinion that women who judge other women badly are just '(...) jealous of those who are at ease' (M1_sg8), probably because they also wanted to feel free to engage in casual relationships but don't feel confident to do so. One female participant went even further and added that 'For instance, if a person is a slut, they may say that she's a slut but she's going to continue to have sex and she's the one that is going to laugh. While everyone is insulting her, she's going to continue to have sex...' (W1_mg4). During the interviews, women referred to men engaging in these depreciative judgments towards permissive women as well, but male participants never actually made such an assertion, instead only affirming what they believe is society's general thinking about women's sexual behavior.

As a result of all the criticism, 'Girls only talk to certain people that they know are not going to judge them' (W2_sg6). They rely on a circle of trusted friends, where they feel protected and supported, a safe place where they have no fear of being socially reproved. Women also share their experiences with men as long as they have no sexual or romantic interest in them, 'But that depends to whom we talk about it (...) if we talk among friends, even boys, I don't think that's a problem, but if we want to have some kind of relationship with one of the boys we are telling these things we might feel a bit more inhibited because of what he may think about us' (W1_sg6).

\section{Discussion}

We have argued the focus of the sexual double standard has shifted to target behaviors such as casual sexual relationships. As the attitudes around these behaviors have not been noted or explored in previous Portuguese studies we sought to examine the spontaneous remarks regarding gender sexual standards that young adults made while participating in a larger study about the characteristics of CSRs. These statements were not specifically prompted, which brings additional interest to the analysis.

Findings from this study indicate an ongoing change in the acceptability of the CSRs among Portuguese college students, including those with and without their own experience of CSRs. We note the emergence of a single sexual standard - one that is mostly liberal, but at times also conservative. An increase in the proportion of single individuals and the fulfillment of their sexual and emotional needs - namely through their sexual relationships - does not, however, occur without social resistance. The challenging of normative gender roles is culture-specific and may include struggles for women to be perceived as personally and socially complete despite not being married (Himawan et al., 2018), for the right to premarital sex (Zheng et al., 2011), or for the use of the same standard in accepting sexual involvement in noncommitted relationships (Endendijk et al., 2020). It is this last aspect that is at issue in the culture in which the present study is embedded, specifically in the acceptance of CSR for women and not only for men (Amaro et al., 2021; Neves, 2016). Thus, if our study points, on the one hand, to egalitarian positions, on the other there remain some inequalities that derive from a deep-rooted traditional SDS still recognized in Portuguese society by the younger generations. It is not clear whether participants have internalized these beliefs from the previous generations, but the social existence of the standard is highlighted, and the cultural and religious background in which these youngsters grew up may help explain the clashes between traditional and liberal beliefs. Liberal beliefs regarding sex and relationships, in line with contemporary social discourses valuing gender equality, stand alongside a still-existing conservative religious heritage. The coexistence of liberal and traditional standards has already been found in other studies (e.g., Alarcão et al., 2015; Reid et al., 2011) and in meta-analyses on the SDS (e.g., Endendijk et al., 2020; Petersen \& Hyde, 2011), which suggests a relative independence between the normality of the conduct and the norm used to evaluate it (Amaro et al., 2021). This is still more likely as previous Portuguese studies have pointed to the emergence of the SDS more as a perceived social shared belief than a personally endorsed sexual standard (Marques et al., 2013; Ramos et al., 2005). This is to say that liberal positions, more so than conservative ones, 
tend to be adopted despite recognition of the social SDS. However, references to the consequences of the traditional SDS help to explain how its social existence contributes to the perpetuation of dual judgments and behaviors, independently of an individual's self-identification with such a standard (Amaro et al., 2021).

One important finding is the recognition among these young adults that female and male sexuality are derived from the same biological imperatives - and, thus, male and female sexuality can both be equally accepted by society. Participants believe women are entitled to seek pleasure, to have the same sexual freedom as men, and to feel confident in proactively looking for casual relationships. In particular, the young women we interviewed advocate not for more acceptance of their behaviors, but simply that the standards be applied equally to both genders. This may be explained by the fact that these participants did not have a particularly strong religious affiliation, and certainly did not endorse orthodox religious beliefs, as those would probably impose a more rigorous focus on abstinence and prevent them from understanding sexual drive as a biological instinct (Himawan, 2020).

Somewhat less expected was the easiness reported by women in our sample regarding initiating and leading the CSRs, contrary to what has been found in the literature (e.g., Sakaluk et al., 2014). In the current study, we found that the more confident or experienced partner is usually the one responsible for taking the leading role. Furthermore, women bringing condoms, a behavior which suggests an expectation of sexual intercourse, does not seem to violate the subtle sexual double standard related to sexual permissiveness in our sample, as it once did (Hynei \& Lydon, 1995). These proactive, agential actions show that women do not have to wait for men to make the first move and feel perfectly at ease to take the lead themselves, which points to the weakening of traditional female gender roles and the blurring of their differences from traditional male roles.

Results also indicate that there appears to be a specific time and place to engage in CSRs, which helps to normalize and de-penalize the behavior. Such a consideration is reflected in both men's and women's desire to have committed relationships in the future, as also found in other cultures (e.g., Garcia \& Reiber, 2008; Himawan et al., 2018). Likewise, it is expressed in the idea that certain contextual factors attenuate the consequences of the social judgment around CSRs, such as moving to a new town when going to college. These young adults stressed the liberating effect of anonymity, especially for women. In such favorable contexts, women can free themselves from the SDS that puts them under moral constraint and prevents them from acting according to their desires (Reid et al., 2011).

It is important to note that there was some convergence on the limits, difficulties, and challenges encountered by both genders when dealing with CSRs. Women are reproached for having too many partners, but this also poses a threat to men's reputations and jeopardizes future (committed) relationships, since women affirm they tend not to trust men who are promiscuous. This points to an increasingly egalitarian conservative judgment of these sexual behaviors, as found in previous research (Allison \& Risman, 2013), consistent with the less-permissive sexual codes perceived among participants' parents' generation.

Although we found both liberal and conservative gender equality, women are judged more negatively based on the number of sexual partners than men are, as observed in the literature (Marks et al., 2019). We found women still suffering more criticism than men do, especially criticism expressed by other women, as also found elsewhere (Farvid et al., 2017). Such criticism targets not only the number of partners but also their succession without a proper waiting period. It has been proposed in the literature that women try to make a clear distinction between themselves and other women who appear to have erotic natures that they believe are not appreciated by men (Hamilton, 2007), criticism being a weapon that allows them to depict how different (and thus, more desirable) they are. As such, women in our study only confide these experiences to close friends, who they know are not going to vilify them.

It can be said that society's constraints concerning the SDS affect both men and women - e.g., inhibition of female initiative and alcohol-assisted dis-inhibition of male initiative, in order to prove or protect one's social image and sexual reputation - although it affects women more severely and results in different consequences for each gender. It is possible that, resulting from visible gender equality in access to higher education, career building, and the labor market (Himawan, 2020), and being therefore more asserted and established in society, the social pressure on sexual behavior has been shifted to controlling men's behavior as a way to maintain the status quo of oppression around women's sexuality.

Nevertheless, contrary to what has been found in national (e.g., Marques et al., 2013) and international literature (e.g., Holland \& Vangelisti, 2020; Petersen \& Hyde, 2010), the young men interviewed in this study, whether in mixed or homogenous groups, did not endorse a traditional SDS for CSRs, but rather expressed some outrage over its existence in society. Being part of a population with university attendance is usually associated with more permissiveness (e.g., Laumann et al., 2000), and the similar attitudes towards postponing marriage and remaining single (Himawan et al., 2018) help to understand this egalitarian expression. While there is hope that these younger generations are indeed more open-minded, the gender of the interviewer that led the focus groups (female) and an impression management effect may have contributed to advancing this idea. 
Our results also indicate that despite being criticized, some women rely on their own strength and continue to enjoy their sexuality, ignoring the envy and unkindness that underlies some judgments. This proactive role is even more important in view of the tension resulting from women's willingness to experience pleasure, to go and get want they want, vs. the social criticisms of women's involvement in CSRs. The jealousy some women feel toward those who have the capacity not to care about what is said indicates that, for some, the involvement in CSRs is probably not an internal conflict; it is a struggle against external and social forces, one that can create empowerment. This seems to result also in admiration of women who have managed to evade the restrictions that the SDS intends to put on their behavior.

In a nutshell, participants expressed positive attitudes towards CSRs, while their spontaneous discourse about the role of gender in these particular sexual relationships focused on equality and, at the same time, on the shared perception that society evaluates men more positively and women more negatively for being involved in CSRs or with multiple partners. While the simultaneous presence of references to egalitarian, liberal, and differential sexual standards is not necessarily new, the fact that women claim active sexual roles for themselves, even defending the right to extol their sexuality, and the fact that men are especially critical of the SDS, in a society in which this traditional standard appears to remain deeply-rooted, are unique in the panorama of Portuguese research. Equally surprising is the reference to a conservative egalitarian standard, which seems to reveal the values of previous generations, but which does not exclude the acceptance of CSRs, as long as certain limits on space, time, and number of partners are observed. Independently of being more or less expected, these various discourses, because they come from contrasting positions, also point to an apparent lag in the way social transformations related to the normality and normativity of casual sexual relationships are taking place. On the one hand, we have the adoption of liberal sexual standards that seem to reflect the social changes resulting from the self-development imperative (Hamilton \& Armstrong, 2009) that is spreading across cultures (Himawan et al., 2018). Being afforded distance from any urgency toward marriage and family life, such liberal standards welcome new forms of relationships, including casual sexual relationships. On the other hand, these transformations do not seem to be reflected in the social norms that preside over the acceptance and judgment of casual sexual involvement, given the still-entrenched SDS recognized in Portuguese society. This is all the more noteworthy insofar as those who dare not go against its prescriptions, for fear of the negative repercussions on their sexual image and reputation, may end up limiting their access to positive, free, and satisfying sexual experience.

\section{Limitations and Future Directions}

The current study has limitations that must be addressed. First, the fact that there has been no explicit objective for the study of the SDS in CSRs has certainly left many aspects to be investigated and has contributed to a limited view of the theme. Even though the SDS is found more in qualitative studies than in laboratory-based experimental research (Marks et al., 2019), in the present study the reports were mixed. Qualitative studies are considered to have a higher degree of ecological validity and, as such, the fact that specific questions concerning any particular exploration of the topic were not asked may bring considerable validity especially to the egalitarian judgments expressed regarding the CSRs. Second, due to the homogeneity of the sample, results may only be applicable to college students and heterosexual individuals, as only students enrolled in a university were invited to participate and the sample size of non-heterosexual individuals was small. Further studies to deepen our understanding of the attitudes toward CSRs encountered in this study are needed to help to disentangle their idiosyncrasy from changes taking place more widely, to clarify their implications for gender equality and their consequences for the lived experience of sexuality, in particular of sexual satisfaction.

\section{Conclusions}

Involvement in CSRs, especially frequent involvement, is considered "a barometer of the degree to which attitudes toward sexuality have continued to liberalize (or not) in recent decades" (Alisson \& Risman, 2013, p. 1192). In our study, however, more- and less-liberal attitudes coexist, as reflected in the recognition of the simultaneous presence of traditional gendered sexual standards and egalitarian ones, or the apparent rejection of the former and endorsement of the latter (in forms more liberal than conservative). Such a multifaceted reality has consequences for both men and women. While the traditional SDS highlights the social benefits of casual sex for men to the point of pressuring them to engage in CSRs, an egalitarian conservative standard penalizes men (not only women) who have a high number of sexual partners, rendering them less desirable for the steady relationships that are their long-term goals. For women, the existence of concomitant discourses means that some of them refrain from taking the initiative in these types of relationships, as well as hold back from disclosing their casual sexual experiences to others, while others live their sexual lives freely without regard for social judgment.

Even if challenging the normative gender roles occurs cross-culturally, in the present study we found that the normalization of CSRs seems to belong mainly to women 
who do not share the existing norm or who resist internalizing it, placing the conflict in social space and using their behavior as a weapon to combat the usual normative judgment. These agentic women appear to be the ones helping to normalize needs, desires, and sexual behaviors in CSRs, which are perceived under the rubric of a liberal SSS. This is aided by the position of men who do not express a traditional SDS in CSRs and who show some outrage at its existence in society. Such challenges to the norm are a way of changing social constraints - especially in relation to the experience of CSRs - which seems to be desired by many women and defended by men. This is identified and admired in some of the most agentic women, whose striving contributes to the normalization and more equal evaluation of this conduct.

Supplementary Information The online version contains supplementary material available at https://doi.org/10.1007/s12144-021-02344-9.

Funding This work was supported by the Portuguese Science Foundation (FCT), under Grant (number PTDC/PSI-GER/28530/2017). This work received national funding from FCT - Fundação para a Ciência e a Tecnologia, I.P, through the Research Center for Psychological Science of the Faculty of Psychology, University of Lisbon (UIDB/04527/2020; UIDP/04527/2020).

Data Availability data are available upon request to the first author. Conflict of interest/Competing interests.

On behalf of all authors, the corresponding author states that there is no conflict of interest.

\section{Declarations}

Ethical Approval All procedures performed in studies involving human participants were in accordance with the ethical standards of the institutional and/or national research committee and with the 1964 Helsinki declaration and its later amendments or comparable ethical standards.

Informed Consent Informed consent was obtained from all individual participants included in the study.

\section{References}

Aboim, S. (2005). A formação do casal: Formas de entrada e percursos conjugais [The formation of the couple: Ways of entry and marital pathways]. In K, Wall. (Eds.), Famílias em Portugal - Percursos, Interaçções, Relações Sociais (pp. 85-116). Imprensa de Ciências Sociais.

Alarcão, V., Virgolino, A., Roxo, L., Machado, F. L., \& Giami, A. (2015). Exploring gender in Portuguese bedrooms: Men's and women's narratives of their Sexuality through a mixed methods approach. Sociological Research Online, 20(2), 8. https://doi.org/ 10.5153/sro.3619

Allison, R., \& Risman, B. J. (2013). A double standard for 'Hooking Up': How far have we come toward gender equality? Social Science Research, 42(5), 1191-1206.
Alvarez, M.-J., Garcia, M., \& Pereira, C. (2019). A diversidade dos relacionamentos casuais e suas características [The diversity of casual relationships and their characteristics]. Psicologia, 33(2), 9-26.

Alvarez, M.-J., Pereira, C. R., Godinho, C. A., \& Luz, R. (2021). Clearcut terms and culture-sensitive characteristics of distinctive casual sexual relationships in Portuguese emerging adults. Sexuality \& Culture. Advance online publication https://link.springer.com/ article/https://doi.org/10.1007/s12119-021-09859-0

Amaro, H., Alvarez, M.-J., \& Ferreira, J. A. (2021). Portuguese college students' perceptions about the social sexual double standard: Developing a comprehensive model for social SDS. Sexuality \& Culture, 25, 733-755.

Arnett, J. J. (2015). Emerging adulthood: The winding road from the late teens through the twenties ( $2^{\text {nd }}$ ed.). Oxford Press.

Bordini, G. S., \& Sperb, T. M. (2013). Sexual Double Standard: A review of the literature between 2001 and 2010. Sexuality \& Culture, 17(4), 689-704.

Braun, V., \& Clarke, V. (2006). Using thematic analysis in psychology. Qualitative Research in Psychology, 3(2), 77-101.

Eagly, A. H., \& Wood, W. (1999). The origins of sex differences in human behavior: Evolved dispositions versus social roles. American Psychologist, 54(6), 408-423.

Eaton, A., Rose, S., Interligi, C., Fernandez, K., \& McHugh, M. (2016). Gender and ethnicity in dating, hanging out, and hooking up: Sexual scripts among Hispanic and white young adults. Journal of Sex Research, 53(7), 788-804.

Endendijk, J. J., van Baar, A. L., \& Dekovic, M. (2020). He is a stud, she is a slut! A meta-analysis on the continued existence of Sexual Double Standards. Personality and Social Psychology Review, 24(2), 163-190.

England, P., \& Bereak, J. (2014). The sexual double standard and gender differences in attitudes toward casual sex among U. S. university students. Demographic Research, 30, art.46, 1327-1338.

Farvid, P., Braun, V., \& Rowney, C. (2017). 'No girl wants to be called a slut!': Women, heterosexual casual sex and the sexual double standard. Journal of Gender Studies, 26(5), 544-560.

Ferreira, P. M., \& Villaverde Cabral, M. (Eds.) (2010). Sexualidades em Portugal: Comportamentos e riscos [Sexualities in Portugal: Behaviors and risks]. Bizâncio.

Fjaer, E. G., Pedersen, W., \& Sandberg, S. (2015). 'I'm not one of those girls': Boundary-work and the sexual double standard in a liberal hookup context. Gender \& Society, 29(6), 960-981.

Frith, H. (2000). Focusing on sex: Using focus groups in sex research. Sexualities, 3, 275-297.

Garcia, J. R., \& Reiber, C. (2008). Hook-up behavior: A biopsychosocial perspective. Journal of Social Evolutionary and Cultural Psychology, 2(4), 192-208.

Garcia, J. R., Reiber, C., Massey, S., \& Merriwether, A. (2012). Sexual hookup culture: A review. Review of General Psychology, 16(2), 161-176.

Hamilton, L. (2007). Trading on heterosexuality: College women's gender strategies and homophobia. Gender \& Society, 21(2), $145-172$.

Hamilton, L., \& Armstrong, E. (2009). Gender sexuality in young adulthood: Double binds and flawed options. Gender \& Society, 23(5), 589-616.

Himawan, K. (2020). Singleness, sex, and spirituality: How religion affects the experience of being single in Indonesia. Mental Health, Religion \& Culture, 23(2), 204-215.

Himawan, K., Bambling, M., \& Edirippulige, S. (2018). The Asian single profiles: Discovering many faces of never married adults in Asia. Journal of Family Issues, 39(14), 3667-3689.

Holland, M., \& Vangelisti, A. L. (2020). The sexual double standard and topic avoidance in friendships. Communication Quarterly, 68(3), 306-330. 
Hyde, J. S. (2005). The gender similarities hypothesis. American Psychologist, 60(6), 581-592.

Hynei, M., \& Lydon, J. E. (1995). Women's perceptions of female contraceptive behavior: Experimental evidence of the sexual double standard. Psychology of Women Quarterly, 19(4), 563-581.

Kennair, L. E., Wyckoff, J. P., Asao, K., Buss, D. M., \& Bendixen, M. (2018). Why do women regret casual sex more than men do? Personality and Individual Differences, 127, 61-67.

Laumann, E. O., Gagnon, J. H., Michael, R. T., \& Michaels, S. (2000). The social organization of sexuality: Sexual practices in the United States. University of Chicago Press.

Lyons, H., Manning, W., Longmore, M., \& Giordano, P. (2014). Young adult casual sexual behavior life-course-specific motivations and consequences. Sociological Perspectives, 57(1), 79-101.

Machado, M. C., Alves, M. I., Couceiro, L., Silva, F., Morais de Almeida, M., \& Alves, I. (2014). Natalidade e fertilidade: Análise dos conhecimentos e expectativas de 3585 estudantes universitários portugueses [Birth and fertility: Analysis of the knowledge and expectations of 3585 Portuguese university students]. Acta Médica Portuguesa, 27(5), 601-608.

Marks, M. J., Young, T. M., \& Zaikmen, Y. (2019). The sexual double standard in the real world. Social Psychology, 50(2), 67-79.

Marques, N., Vieira, R., \& Pechorro, P. (2013). The sexual double standard in a masculine way: A Portuguese transgenerational perspective. Revista Internacional De Andrologia, 11(4), 138-143.

National Institute of Population and Social Security Research (2017). The fifteenth Japanese national fertility survey in 2015. Marriage process and fertility of married couples attitudes toward marriage and family among Japanese singles. Highlights of the survey results on married couples/singles.

Neves, D. M. (2016). Normas face ao género e à diversidade sexual: mudanças inacabadas nos discursos juvenis [Gender and sexual diversity norms: Ongoing changes in youth discourses]. Sociologia, Problemas e Práticas [Online], 82, retrieved June 22, 2021. URL: http://journals.openedition.org/spp/2506

Olmstead, S., Norona, J., \& Anders, K. (2019). How do college experience and gender differentiate the enactment of hookup scripts among emerging adults? Archives of Sexual Behavior, 48, $1769-1783$.

Papp, L. J., Hagerman, C., Gnoleba, M. A., Erchull, M. J., Liss, M., Miles-McLean, H., \& Robertson, C. M. (2015). Exploring perceptions of slut-shaming on Facebook: Evidence for a reverse sexual double standard. Gender Issues, 32(1), 57-76.

Petersen, J. L., \& Hyde, J. S. (2010). A meta-analytic review of research on gender differences in sexuality: 1993-2007. Psychological Bulletin, 136(1), 21-38.
Petersen, J. L., \& Hyde, J. S. (2011). Gender differences in sexual attitudes and behaviors: A review of meta-analytic results and large datasets. Journal of Sex Research, 48(2-3), 149-165.

PORDATA - Base de dados Portugal contemporâneo. Educação: Escolaridade da população [Education: Educational attainment levels in the population]. Retrieved May 20, $2021 \mathrm{https} / / \mathrm{www}$. pordata.pt/Portugal/Popula\%c3\%a7\%c3\%a3o+residente+com+ $15+\mathrm{e}+$ mais + anos + de + idade + por $+\mathrm{n} \% \mathrm{c} 3 \%$ advel + de + escolarida de+completo+mais+elevado+total+e+por+sexo-333

Ramos, V., Carvalho, C. C., \& Leal, I. P. (2005). Atitudes e comportamentos sexuais de mulheres universitárias: A hipótese do duplo padrão [Sexual attitudes and behaviors among women college students: The hypothesis of sexual double standard]. Análise Psicológica, 23(2), 173-185.

Reid, J. A., Elliott, S., \& Webber, G. R. (2011). Casual hookups to formal dates: Refining the boundaries of the sexual double standard. Gender \& Society, 25(5), 545-568.

Rudman, L. A., Glick, P., \& Marquardt, T. (2017). When women are urged to have casual sex more than men are: Perceived risk moderates the sexual advice double standard. Sex Roles, 77(5-6), 409-418.

Sakaluk, J. K., Todd, L. M., Milhausen, R., \& Lachowsky, N. J. (2014). Dominant heterosexual sexual scripts in emerging adulthood: Conceptualization and measurement. Journal of Sex Research, 51(5), 516-531.

Sprecher, S., Treger, S., \& Salaluk, J. K. (2013). Premarital sexual standards and sociosexuality: Gender, ethnicity, and cohort differences. Archives of Sexual Behavior, 42(8), 1395-1405.

Tong, A., Sainsbury, P., \& Craig, J. (2007). Consolidated criteria for reporting qualitative research (COREQ): A 32-item checklist for interviews and focus group. International Journal of Quality in Health Care, 19(6), 349-357.

Wentland, J., \& Reissing, E. (2011). Taking casual sex not too casually: Exploring definitions of casual sexual relationships. Canadian Journal of Human Sexuality, 20(3), 75-91.

Wood, W., \& Eagly, A. H. (2012). Biosocial construction of sex differences and similarities in behavior. In J. M. Olson \& M. P. Zanna (Eds.), Advances in Experimental Social Psychology (Vol. 46, pp. 55-123). Academic Press.

Zheng, W., Zhou, X., Zhou, C., Liu, W., \& Hesketh, T. (2011). Detraditionalisation and attitudes to sex outside marriage in China. Culture, Health \& Sexuality, 13(5), 497-511.

Publisher's note Springer Nature remains neutral with regard to jurisdictional claims in published maps and institutional affiliations. 